Pacific

Journal of

Mathematics

THE INDUCED WEIL REPRESENTATION AND THE SHALIKA PERIOD

Zhengyu Mao and Stephen Rallis

Volume $191 \quad$ No. 2

December 1999 


\title{
THE INDUCED WEIL REPRESENTATION AND THE SHALIKA PERIOD
}

\author{
Zhengyu Mao and Stephen Rallis
}

The relative trace formula method can be used to prove the lifting of cuspidal representations of $G S p_{2}$ to $G L_{4}$. We prove the fundamental lemma for the relative trace formula using the induced Weil representation.

\section{Introduction.}

In $[\mathbf{F}-\mathbf{J}]$, Friedberg and Jacquet introduce a relative trace formula which gives the lifting from the cuspidal representations of $G S p_{2}$ to the selfcontragradient cuspidal representations of $G L_{4}$. In this paper, we prove the fundamental lemma for this trace formula, meanwhile illustrate the method of using Weil representation in computing local integrals. What we emphasize here is the method of the proof. It can be applied to show fundamental lemma for many other relative trace formulas, which are out of the scope of previously available methods.

(1.1) Let $F$ be a number field, $\mathbf{A}$ its adele ring. Let $\tau_{n}$ be a $n \times n$ matrix with 1's on the antidiagonal and 0's elsewhere. The group $G S p_{2}(F)$ is the similitude group associated to the symplectic form $w_{0}=\left[\begin{array}{cc}\tau_{2} \\ -\tau_{2}\end{array}\right]$. The Shalika subgroup $H$ of $G L_{4}(F)$ is the set

$$
H=\left\{\left[\begin{array}{ll}
h & \\
& h
\end{array}\right]\left[\begin{array}{cc}
I & \\
X & I
\end{array}\right] \mid h \in G L_{2}(F), X \in M_{2,2}(F)\right\} .
$$

Let $\psi$ be a nontrivial additive character of $\mathbf{A} / F, \chi$ a multiplicative character of $\mathbf{A}^{\times} / F^{\times}$. Define a character $\Xi$ on $H$ by

$$
\Xi\left(\left[\begin{array}{ll}
h & \\
& h
\end{array}\right]\left[\begin{array}{cc}
I & \\
X & I
\end{array}\right]\right)=\chi^{-1}(\operatorname{det} h) \psi(\operatorname{tr}(X)) .
$$

A cuspidal representation $\Pi$ of $G L_{4}(\mathbf{A})$ is said to be $(H, \Xi)$ distinguished if the central character of $\Pi$ equals $\chi^{2}$, and there is $\phi \in \Pi$ with its Shalika period ( $Z$ being the center of $H$ )

$$
\int_{Z(\mathbf{A}) H(F) \backslash H(\mathbf{A})} \phi(h) \Xi(h) d h \neq 0 .
$$


It follows from $[\mathbf{J}-\mathbf{S}]$ that the twisted exterior square $L$-function $L\left(s, \Pi, \wedge^{2} \otimes\right.$ $\left.\chi^{-1}\right)$ has a pole at $s=1$ if and only if $\Pi$ is $(H, \Xi)$ distinguished. Meanwhile, from the philosophy of $L$-groups, the existence of the pole at $s=1$ implies that $\Pi$ comes from a functorial lifting from $G S p_{2}(F)$. One tries to verify this implication using the relative trace formula. This work is started in $[\mathbf{F}-\mathbf{J}]$, see there for an excellent introduction to the problems we consider.

(1.2) We now state the relative trace formula in $[\mathbf{F}-\mathbf{J}]$. Let $N$ and $N^{\prime}$ be the group of upper triangular unipotent matrices in $G L_{4}$ and $G S p_{2}$ respectively. For $f \in C_{c}^{\infty}\left(G L_{4}(\mathbf{A})\right)$ and $f^{\prime} \in C_{c}^{\infty}\left(G S p_{2}(\mathbf{A})\right)$, define the kernel functions:

$$
\begin{aligned}
K_{f}(x, y) & =\sum_{\gamma \in G L_{4}(F)} f\left(x^{-1} \gamma y\right), x, y \in G L_{4}(\mathbf{A}), \\
K_{f^{\prime}}(x, y) & =\sum_{\gamma \in G S p_{2}(F)} f^{\prime}\left(x^{-1} \gamma y\right), x, y \in G S p_{2}(\mathbf{A}) .
\end{aligned}
$$

The relative trace formula is the following claim: There is a map $\epsilon$ from $C_{c}^{\infty}\left(G L_{4}(\mathbf{A})\right)$ to $C_{c}^{\infty}\left(G S p_{2}(\mathbf{A})\right)$, at almost all places extending a Hecke algebra homomorphism $(\S 4)$, such that we have an identity of distributions $J(\epsilon(f))=I(f)$, where

$$
\begin{aligned}
I(f) & =\iint K_{f}\left(h^{-1}, n\right) \Xi(h) \theta(n) d n d h, \\
J\left(f^{\prime}\right) & =\iiint K_{f^{\prime}}\left({ }^{t} n_{1}, n_{2} \zeta\right) \theta^{\prime}\left(n_{1}^{-1} n_{2}\right) \chi(\zeta) d n_{1} d n_{2} d^{\times} \zeta .
\end{aligned}
$$

Here $h$ is integrated over the Shalika subgroup $H(F) \backslash H(\mathbf{A}), n$ is integrated over $N(F) \backslash N(\mathbf{A}), n_{1}, n_{2}$ are integrated over $N^{\prime}(F) \backslash N^{\prime}(\mathbf{A}), \zeta$ is integrated over $F^{\times} \backslash \mathbf{A}^{\times}$; the characters $\theta, \theta^{\prime}$ are nondegenerate, and are defined as follows: For $n \in N(\mathbf{A}), \theta(n)=\psi\left(n_{12}+n_{23}+n_{34}\right)$, for $n \in N^{\prime}(\mathbf{A}), \theta^{\prime}(n)=$ $\psi\left(n_{12}+n_{23}\right)$. (We use $g_{i j}$ to denote the entry of a matrix $g$ on the $i$-th row and $j$-th column.)

Note in the spectral decomposition of $I(f)$, only the $(H, \Xi)$ distinguished representations appear. The above trace formula should lead to a correspondence between automorphic representations of $G S p_{2}$ and the $(H, \Xi)$ distinguished automorphic representations of $G L_{4}$. Moreover, it would also imply that every automorphic representation of $G S p_{2}$ whose functorial lift to $G L_{4}$ is cuspidal will be near equivalent to a generic representation, (one with nontrivial Whittaker model).

(1.3) We can unwind the integrals (2) and (3) into sums of orbital integrals. This is done in $[\mathbf{F}-\mathbf{J}]$. Let $w$ be a permutation matrix in $G L_{4}$, a be a diagonal matrix in $G L_{4}$. At any local place $v$, for $f_{v} \in C_{c}^{\infty}\left(G L_{4}\left(F_{v}\right)\right)$, 
define the local orbital integral

$$
I_{f_{v}}(w, \mathbf{a})=\int_{N_{v} \cap w^{-1} H_{v} w \backslash N_{v}} \int_{H_{v}} f_{v}(h w \mathbf{a} n) \Xi(h) \theta(n) d n d h .
$$

Fix the Haar measures so that $\mathbf{A} / F$ has volume 1 . Then for $f=\otimes f_{v}$, we have

$$
I(f)=\sum_{(w, \mathbf{a})} \prod_{v} I_{f_{v}}(w, \mathbf{a})
$$

where the sum is taken over $(w, \mathbf{a})$ of the following form:

(1) $w=(12), \mathbf{a}=\operatorname{diag}\left[a_{1} a_{2}, a_{1}, 1,1\right]$.

(2) $w=1, \mathbf{a}=\operatorname{diag}\left[-a_{1}, a_{1}, 1,1\right]$.

(3) $w=(132), \mathbf{a}=\operatorname{diag}\left[a_{2}, 1,1,1\right]$.

(4) $w=(1243), \mathbf{a}=1$.

Similarly, for $f_{v}^{\prime} \in C_{c}^{\infty}\left(G S p_{2}\left(F_{v}\right)\right)$, define

$$
J_{f_{v}^{\prime}}\left(w^{\prime}, \mathbf{t}\right)=\int_{N_{v}^{\prime}} \int_{N_{v}^{\prime} \cap w^{\prime-1} \bar{N}_{v}^{\prime} w^{\prime} \backslash N_{v}^{\prime}} \int_{F_{v}^{\times}} f^{\prime}\left({ }^{t} n_{2} w^{\prime} \mathbf{t} n_{1} \zeta\right) \chi(\zeta) \theta^{\prime}\left(n_{1} n_{2}\right) d n_{1} d n_{2} d \zeta
$$

where $\bar{N}^{\prime}$ is the opposite of $N^{\prime}, w^{\prime}$ is a Weyl element in $G S p_{2}$, and $\mathbf{t}$ is a diagonal matrix $G S p_{2}$. Then for $f^{\prime}=\otimes f_{v}^{\prime}$, we have

$$
J\left(f^{\prime}\right)=\sum_{\left(w^{\prime}, \mathbf{t}\right)} \prod_{v} J_{f_{v}^{\prime}}\left(w^{\prime}, \mathbf{t}\right)
$$

where the sum is taken over $\left(w^{\prime}, \mathbf{t}\right)$ of the form:

$\left(1^{\prime}\right) w^{\prime}=1, \mathbf{t}=\operatorname{diag}\left[-a_{1} a_{2},-a_{2}, 1, a_{1}^{-1}\right]$.

$\left(2^{\prime}\right) w^{\prime}=w_{1}, \mathbf{t}=\operatorname{diag}\left[a_{1}, 1,-1,-a_{1}^{-1}\right]$.

$\left(3^{\prime}\right) w^{\prime}=w_{2}, \mathbf{t}=\operatorname{diag}\left[a_{2}, a_{2}, 1,1\right]$.

$\left(4^{\prime}\right) w^{\prime}=w_{0}, \mathbf{t}=1$.

For the notations, see [F-J, §I.2-3]. We say $(w, \mathbf{a})$ and $\left(w^{\prime}, \mathbf{t}\right)$ match if they are of the form $(i)$ and $\left(i^{\prime}\right), i=1,2,3$ or 4 , for the same $a_{1}, a_{2}$. To prove the claim in (1.2), one needs to show the existence of a map $\epsilon$, such that if $f_{v}^{\prime}=\epsilon\left(f_{v}\right)$, we have

$$
I_{f_{v}}(w, \mathbf{a})=\Delta_{v}(w, \mathbf{a}) J_{f_{v}^{\prime}}\left(w^{\prime}, \mathbf{t}\right)
$$

for all matching $(w, \mathbf{a})$ and $\left(w^{\prime}, \mathbf{t}\right)$, and $\Delta_{v}$ is a transfer factor satisfying

$$
\prod_{v} \Delta_{v}(w, \mathbf{a}) \equiv 1
$$

(1.4) One of the main steps in proving the identity $(*)$ is to show the fundamental lemma. From now on, let $F_{v}$ be a local non-Archimedean field, with odd residue characteristic. We will drop the reference to the place $v$ in the notations. Let $\mathcal{O}$ be the ring of integers in $F$, let $K=G L_{4}(\mathcal{O})$, $K^{\prime}=G S p_{2}(\mathcal{O}), K^{\prime \prime}=G O_{6}(\mathcal{O})$. A Hecke function of $G L_{4}$ is a bi- $K$-invariant 
compactly supported function on $G L_{4}(F)$. Let $\mathcal{H}\left(G L_{4} / / K\right)$ be the algebra of Hecke functions. Similarly we define the Hecke algebras $\mathcal{H}\left(G S p_{2} / / K^{\prime}\right)$ of $G S p_{2}$, and $\mathcal{H}\left(G O_{6} / / K^{\prime \prime}\right)$ of $G O_{6}$.

Let $f, f^{\prime}$ be Hecke functions on $G L_{4}$ and $G S p_{2}$ respectively. There is a concept of matching between Hecke functions on $G L_{4}$ and $G S p_{2}$, (see $\S 4$ ). The fundamental lemma asserts that for matching Hecke functions $f$ and $f^{\prime}$, the identity $(*)$ holds. With the assumption that a version of Howe duality conjecture holds for the pair $\left(G O_{6}, G S p_{2}\right)$, we prove:

Theorem 1. If $\psi$ is of order $0, \chi$ is unramified, then

$$
\chi(\operatorname{det}(w \mathbf{a}))^{-1 / 2} I_{f}(w, \mathbf{a})=\chi\left(\lambda\left(w^{\prime} \mathbf{t}\right)\right)^{1 / 2} J_{f^{\prime}}\left(w^{\prime}, \mathbf{t}\right)
$$

for matching Hecke functions $f, f^{\prime}$ and matching $(w, \mathbf{a}),\left(w^{\prime}, \mathbf{t}\right)$.

Here $\lambda$ is the similitude ratio $(\S 2)$.

The identity (6) between the orbital integrals is the fundamental lemma for the case at hand. The Howe duality we use is stated in $\S 4$. Though its proof has yet to be published, it is believed to be known. In the similar case of $\left(O_{2 n}, S p_{n}\right)$, the Howe duality is proved in [R], [Wa] etc.

(1.5) The Main Theorem of $[\mathbf{F}-\mathbf{J}]$ is the above identity for the case $f, f^{\prime}$ being the characteristic functions of $K, K^{\prime}$. The method of the proof there is using Mellin transform, and is technically very difficult. Only with the masterful skill of the authors, the proof is carried through. Our method is using the (induced) Weil representation and Howe duality. (To prove the result for the characteristic functions of $K, K^{\prime}$, one does not need Howe duality, as the equation (19) does hold in this case.)

The induced Weil representation $\Omega$ is a representation of $G O_{6} \times G S p_{2}$. To use the induced Weil representation, we embed $G L_{4}$ into $G O_{6}$. Our idea is to express the local orbital integrals $I_{f}(w, \mathbf{a})$ and $J_{f^{\prime}}\left(w^{\prime}, \mathbf{t}\right)$ using the 'orbital integrals' of a function $\Phi$ (same for both $f$ and $f^{\prime} !$ ) in the space of $\Omega$, then use the properties of the Weil representation to compare the integrals. In particular, neither of the integrals need to be computed explicitly, thus the technical difficulties are avoided.

The method of using Weil representation in proving fundamental lemma first appears in $[\mathbf{M}-\mathbf{R}]$. We note that in proving the existence of the Weil representation, one needs to show certain braid relation holds $([\mathbf{W}],[\mathbf{K}-\mathbf{S}])$. The braid relation is essentially an equality of exponential sums. The fundamental lemma is also an equality of exponential sums. What we prove here is roughly the braid relation implies fundamental lemma.

(1.6) The paper is organized as follows. In $\S 2$, we introduce the induced Weil representation. In $\S 3$, we show two key lemmas that relate the local orbital integrals to the Weil representation. In $\S 4$ we prove the Theorem. In $\S 5$, we generalize the Lemmas in $\S 3$; the generalization could be used to compute integrals over $S p_{n}, G S p_{n}$ and $M p_{n}$, the double cover of $S p_{n}$. We 
intend to study the application of these lemmas in other lifting problems in a future paper.

The first author would like to thank the Ohio State University mathematics department and its Mathematics Research Institute for their hospitality during his visit.

\section{The induced Weil representation.}

The induced Weil representation is defined over the group $G O_{6} \times G S p_{2}$. We first recall the well known embedding of $G L_{4}$ in $G O_{6}$.

(2.1) Let $\langle$,$\rangle be a symmetric bilinear form on V \cong F^{6}$, associated to the matrix $\tau_{6}$. Let $G O_{6}(F)$ be the group of all $F$-linear isomorphisms $g$ from $V$ to $V$ such that there exists $\lambda \in F^{\times}$with $\left\langle g x, g x^{\prime}\right\rangle=\lambda\left\langle x, x^{\prime}\right\rangle, x, x^{\prime} \in V$. If $g \in G O_{6}(F)$, then such a $\lambda$ is unique, and will be denoted by $\lambda(g)$.

Let $e_{i}, i=1,2,3,4$ be the standard basis of $F^{4}$. Let $\pm e_{i} \wedge e_{j}, 1 \leq i<j \leq 4$ be the standard basis of $V$, here the sign is - when $i=2, j=4$, and + otherwise. Then the linear action of $G L_{4}$ on $F^{4}$ gives an action of $G L_{4}$ on $V$. This is the exterior square representation of $G L_{4}$; it gives an embedding $G L_{4} \stackrel{j}{\rightarrow} G O_{6}$. Clearly $\lambda(j(g))=\operatorname{det}(g)$.

We consider the image under $j$ of the various subgroups of $G L_{4}$. Let $\left[\begin{array}{ll}h & \\ & h\end{array}\right] \in H$, it is easy to check that the group $\left\{j\left(\left[\begin{array}{ll}h & \\ & h\end{array}\right]\right)\right\}$ stablizes some one dimensional subspaces of $V$. More precisely

$$
j\left(\left[\begin{array}{ll}
h & \\
& h
\end{array}\right]\right)\left(\left[\begin{array}{cc}
0 & 0 \\
0 & 0 \\
0 & 1 \\
0 & -1 \\
0 & 0 \\
1 & 0
\end{array}\right]\right)=\operatorname{det}(h)\left(\left[\begin{array}{cc}
0 & 0 \\
0 & 0 \\
0 & 1 \\
0 & -1 \\
0 & 0 \\
1 & 0
\end{array}\right]\right) \text {. }
$$

For $\left[\begin{array}{ll}I & \\ X & I\end{array}\right] \in H$, with $X=\left[\begin{array}{ll}x_{1} & x_{2} \\ x_{3} & x_{4}\end{array}\right]$, we have

$$
j\left(\left[\begin{array}{ll}
I & \\
X & I
\end{array}\right]\right)=\left[\begin{array}{ccc}
1 & \\
Y & I & \\
-\frac{\langle Y, Y\rangle}{2} & -\tau_{4} Y \tau_{4} & 1
\end{array}\right], Y=\left[x_{2}, x_{4},-x_{1}, x_{3}\right]^{T} .
$$

The restriction of $j$ to $N$ is an isometry between $N$ and $N^{\prime \prime}$, the unit upper triangular group of $G O_{6}$. For the diagonal subgroup of $G L_{4}$, we have,

$$
j\left(\operatorname{diag}\left[a_{1}, a_{2}, a_{3}, a_{4}\right]\right)=\operatorname{diag}\left[a_{1} a_{2}, a_{1} a_{3}, a_{1} a_{4}, a_{2} a_{3}, a_{2} a_{4}, a_{3} a_{4}\right] .
$$

(2.2) We describe the model for the induced Weil representation.

Let $\left(Y,\langle,\rangle_{1}\right)$ be a 4 -dimensional nondegenerate symplectic vector space over $F$, with $\langle,\rangle_{1}$ corresponds to $w_{0}$. Let $G S p_{2}(F)$ be the group of all $F$ linear isomorphisms $g$ from $Y$ to $Y$ such that there exists $\lambda \in F^{\times}$with 
$\left\langle g y, g y^{\prime}\right\rangle_{1}=\lambda\left\langle y, y^{\prime}\right\rangle_{1}, y, y^{\prime} \in Y$. If $g \in G S p_{2}(F)$, then such a $\lambda$ is unique, and will be denoted by $\lambda(g)$. Let $S p_{2}(F)$ be the subgroup of $g \in G S p_{2}(F)$ with $\lambda(g)=1$.

There is an induced Weil representation $\Omega$ defined on $G O_{6}(F) \times G S p_{2}(F)$. It is defined in [PS-S], can be constructed by first extend the usual Weil representation to a subgroup of $G O_{6} \times G S p_{2}$, then compactly induce to the whole group, [Ro].

Let $Y=Y^{+} \oplus Y^{-}$be the polarization of $Y$. Then $\Omega$ acts on the space of Schwartz functions on $V \otimes Y^{+} \oplus F^{\times}$. We will use a $6 \times 2$ matrix to denote an element in $V \otimes Y^{+}$. Denote an element in $G O_{6} \times G S p_{2}$ by $(\alpha, \beta)$ with $\alpha \in G O_{6}$ and $\beta \in G S p_{2}$. Then $\Omega$ has the following properties: Let $Z \in M_{6,2}(F), a \in F^{\times}$,

$$
\Omega(g, 1) \phi(Z, a)=|\lambda(g)|^{-3} \phi\left(g^{-1} Z, a \lambda(g)\right),
$$

$$
\begin{aligned}
\Omega\left(1,\left[\begin{array}{rr}
g & \\
& g^{*}
\end{array}\right]\right) \phi(Z, a) & =\frac{\gamma(1, \psi)}{\gamma\left(\operatorname{det} g^{6}, \psi\right)}|\operatorname{det} g|^{3} \phi(Z g, 1), g^{*}=\tau_{2}{ }^{t} g^{-1} \tau_{2} \\
\Omega\left(1, w_{0}^{-1}\right) \phi(Z, 1) & =(\gamma(1, \psi))^{-12} \int \psi\left(\operatorname{tr}\left({ }^{t} Z^{\prime} \tau_{6} Z\right)\right) \phi\left(Z^{\prime}, 1\right) d Z^{\prime}
\end{aligned}
$$

$$
\Omega\left(1,\left[\begin{array}{ll}
I & \\
& \lambda I
\end{array}\right]\right) \phi(Z, a)=\phi\left(Z, a \lambda^{-1}\right) .
$$

\section{Two key lemmas.}

Starting from this section, we assume $\psi$ is of order $0, \chi$ is unramified. In particular $\gamma(a, \psi)=1$ if the valuation of $a$ is even. Let $\Phi_{0}$ be the characteristic function of $M_{6.2}(\mathcal{O}) \times \mathcal{O}^{\times}$. Let $f_{0}, f_{0}^{\prime}$ be the characteristic functions of $K, K^{\prime}$. For $z \in F$, let $u(z)=\left[\begin{array}{ll}1 & z \\ 0 & 1\end{array}\right]$; for $g \in G L_{2}$, let $t(g)=\left[\begin{array}{ll}g & \\ & g^{*}\end{array}\right] \in S p_{2}$.

With the following lemma, we can express the orbital integral $I_{f}(w, \mathbf{a})$ using the representation $\Omega$.

\section{Lemma 1.}

$$
\int_{H} f_{0}(h g) \Xi(h) d h=\iint|a|^{6} \Omega(j(g), t(u(z))) \Phi_{0}\left(a X_{0}, a^{-2}\right) \chi(a) \psi(-z) d^{\times} a d z
$$

where $X_{0}$ is the matrix in (7).

Proof. Let $F_{1}(g), F_{2}(g)$ be the LHS and RHS of (15). 
Lemma 1.1. $F_{i}\left(\left[\begin{array}{ll}h_{0} & \\ & h_{0}\end{array}\right] g\right)=F_{i}(g) \chi\left(\operatorname{det} h_{0}\right)$, for $h_{0} \in G L_{2}$.

Proof. When $i=1$, the identity follows from a change of variable $h \rightarrow$ $h\left[\begin{array}{ll}h_{0} & \\ & h_{0}\end{array}\right]^{-1}$. When $i=2$, from (7) and (10), $F_{2}\left(\left[\begin{array}{ll}h_{0} & \\ & h_{0}\end{array}\right] g\right)$ equals

$$
\begin{aligned}
& \iint\left|a \operatorname{det} h_{0}^{-1}\right|^{6} \Omega(j(g), t(u(z))) \\
& \cdot \Phi_{0}\left(a\left(\operatorname{det} h_{0}^{-1}\right) X_{0},\left(a \operatorname{det} h_{0}^{-1}\right)^{-2}\right) \psi(-z) \chi(a) d^{\times} a d z .
\end{aligned}
$$

The identity follows from the change of variable $a \rightarrow a \operatorname{det}\left(h_{0}\right)$.

Lemma 1.2. $F_{i}\left(\left[\begin{array}{ll}I & \\ X & I\end{array}\right] g\right)=\psi(\operatorname{tr}(-X)) F_{i}(g), X \in M_{2,2}(F)$.

Proof. When $i=1$, the identity follows from the change of variable $h \rightarrow$ $h\left[\begin{array}{cc}I & \\ -X & I\end{array}\right]$. When $i=2$, use the formula (8) and (10). $F_{2}\left(\left[\begin{array}{cc}I & \\ X & I\end{array}\right] g\right)$ equals

$$
\iint|a|^{6} \Omega(j(g), t(u(z))) \Phi_{0}\left(\left[\begin{array}{cc}
0 & 0 \\
0 & 0 \\
0 & 1 \\
0 & -1 \\
0 & 0 \\
1 & -x_{1}-x_{4}
\end{array}\right], a^{-2}\right) \chi(a) \psi(-z) d^{\times} a d z .
$$

From (12), it is

$$
\iint|a|^{6} \Omega\left(j(g), t\left(u\left(z-x_{1}-x_{4}\right)\right)\right) \Phi_{0}\left(a X_{0}, a^{-2}\right) \chi(a) \psi(-z) d^{\times} a d z .
$$

The Lemma 1.2 follows from a change of variable $z \rightarrow z+x_{1}+x_{4}$.

Lemma 1.3. $F_{i}(g k)=F_{i}(g)$, for $k \in K$.

Proof. It is well known that with our assumptions, the function $\Phi=\Phi_{0}$ satisfies:

$$
\Omega(j(k), 1) \Phi=\Omega\left(1, k^{\prime}\right) \Phi=\Phi, k \in K, k^{\prime} \in K^{\prime} .
$$

The Lemma 1.3 follows from this identity and $K$-invariance of $f_{0}$.

From Lemma 1.1-1.3 and the Iwasawa decomposition, we only need to show (15) for $g=\left[\begin{array}{ll}p & \\ & I\end{array}\right]$ where $p=\left[\begin{array}{ll}1 & \\ x & 1\end{array}\right]\left[\begin{array}{ll}b & \\ & c\end{array}\right]$. We show $F_{1}(g)=$ $F_{2}(g)=1$ if $|c|=|b|=1,|x| \leq 1$, and $F_{i}(g)=0$ otherwise. 
Using (8), (9) and (10), we get $F_{2}(g)$ equals

$$
\iint|a|^{6}|b c|^{-3} \Phi_{0}\left(a\left[\begin{array}{cc}
0 & 0 \\
0 & 0 \\
0 & b^{-1} \\
0 & -c^{-1} \\
0 & -c^{-1} x \\
1 & z
\end{array}\right], b c a^{-2}\right) \chi(a) \psi(-z) d^{\times} a d z .
$$

The integral is

$$
\iint \chi(a) \psi(-z) d z d^{\times} a
$$

with domain being $\left|a^{2}\right|=|b c|, a, a b^{-1}, a c^{-1}, a c^{-1} x, a z \in \mathcal{O}$. This domain is equivalent to $|a|=|b|=|c| \leq 1,|x| \leq 1$ and $|a z| \leq 1$. When $|b|=|c|<1$, the integration over $z$ is 0 . Thus the integral is nonzero only when $|b|=|c|=1$, in which case it clearly equals 1 .

The integral $F_{1}(g)$ is $\int \Xi(h) d h$ with the domain being

$$
\left[\begin{array}{ll}
h & \\
& h
\end{array}\right]\left[\begin{array}{ll}
I & \\
X & I
\end{array}\right]\left[\begin{array}{ll}
p & \\
& I
\end{array}\right] \in K \text {. }
$$

We see over the domain, $h \in G L_{2}(\mathcal{O})$, thus $p \in G L_{2}(\mathcal{O})$. This implies $|b|=|c|=1,|x| \leq 1$. While the above condition is not satisfied, $F_{1}(g)=0$; when it is satisfied, the domain becomes $h \in G L_{2}(\mathcal{O}), X \in M_{2,2}(\mathcal{O})$, thus $F_{1}(g)=1$.

The following Lemma allows us to use the Weil representation to compute the orbital integral $J_{f^{\prime}}\left(w^{\prime}, \mathbf{t}\right)$ on $G S p_{2}$. In $\S 5$, we have a generalization of the Lemma.

\section{Lemma 2.}

$\iiint_{N^{\prime}} f_{0}^{\prime}\left(n^{-1} g\right) \theta^{\prime}(n) d n=\iiint \Omega(1, g) \Phi_{0}\left(\left[\begin{array}{cc}x & z \\ -1 & y \\ 0 & -1 \\ 0 & -1 \\ 0 & 0 \\ 0 & 0\end{array}\right], 1\right) \psi(x+y) d x d y d z$

Proof. Let $F_{1}(g), F_{2}(g)$ be the LHS and RHS of (17).

Lemma 2.1. $F_{i}\left(n_{0} g\right)=F_{i}(g) \theta^{\prime}\left(n_{0}\right)$, for $n \in N^{\prime}$.

Proof. When $i=1$, the identity follows from a change of variable $n \rightarrow n_{0} n$. When $i=2$, let $n_{0}=\left[\begin{array}{cc}I & V \\ & I\end{array}\right] t(u(s))$. Let $Y_{0}$ be the matrix in (17). Note 
that ${ }^{t} Y_{0} \tau_{6} Y_{0}=\left[\begin{array}{ll}0 & 0 \\ 0 & 2\end{array}\right]$. Thus from (11) and (12),

$$
\begin{aligned}
& \Omega\left(1,\left[\begin{array}{cc}
I & V \\
& I
\end{array}\right] t(u(s)) g\right) \Phi_{0}\left(Y_{0}, 1\right) \\
& =\psi\left(\frac{V_{21}}{2}\right) \Omega(1, g) \Phi_{0}\left(\left[\begin{array}{cc}
x & z+x s \\
-1 & y-s \\
0 & -1 \\
0 & -1 \\
0 & 0 \\
0 & 0
\end{array}\right], 1\right) .
\end{aligned}
$$

Since $\theta^{\prime}\left(n_{0}\right)=\psi\left(s+\frac{V_{21}}{2}\right)$, the Lemma 2.1 follows from changes of variables $y \rightarrow y+s, z \rightarrow z-x s$.

Lemma 2.2. $F_{i}(g k)=F_{i}(g)$, for $k \in K^{\prime}$.

Proof. This follows from (16) and $K^{\prime}$-invariance of $f_{0}^{\prime}$.

From Lemma 2.1, 2.2 and the Iwasawa decomposition, we only need to show (17) for $g=\operatorname{diag}\left[a_{1}, a_{2}, a_{2}^{-1} \lambda, a_{1}^{-1} \lambda\right]$. We show for such a $g, F_{1}(g)=$ $F_{2}(g)=1$ if $\left|a_{1}\right|=\left|a_{2}\right|=|\lambda|=1$ and they equal 0 otherwise.

Using (12) and (14), we get

$$
\Omega(1, g) \Phi_{0}\left(Y_{0}, 1\right)=\frac{\gamma(1, \psi)}{\gamma\left(a_{1}^{6} a_{2}^{6}, \psi\right)}\left|a_{1} a_{2}\right|^{3} \Phi_{0}\left(\left[\begin{array}{cc}
a_{1} x & a_{2} z \\
-a_{1} & a_{2} y \\
0 & -a_{2} \\
0 & -a_{2} \\
0 & 0 \\
0 & 0
\end{array}\right], \lambda^{-1}\right) .
$$

The integral $F_{2}(g)$ is

$$
\iiint \frac{\gamma(1, \psi)}{\gamma\left(a_{1}^{6} a_{2}^{6}, \psi\right)}\left|a_{1} a_{2}\right|^{3} \psi(x+y) d x d y d z
$$

with domain being $|\lambda|=1, a_{1}, a_{2}, a_{1} x, a_{2} z, a_{2} y \in \mathcal{O}$. If $\left|a_{1}\right|<1$ or $\left|a_{2}\right|<1$, then the integral over either $x$ or $y$ equals 0 . Thus $F_{2}(g)$ is nonzero only when $|a|=|b|=|\lambda|=1$, in which case it equals 1 . Here we need the fact that $\gamma(a, \psi)=1$ when the valuation of $a$ is even.

The integral $F_{1}(g)$ is $\int \theta^{\prime}(n) d n$ with the domain being $n^{-1} g \in K^{\prime}$. Over the domain, we have $\left|a_{1}\right|=\left|a_{2}\right|=|\lambda|=1$ and $n \in K^{\prime}$. Thus $F_{1}(g)$ is nonzero only when $\left|a_{1}\right|=\left|a_{2}\right|=|\lambda|=1$, in which case the integration over $n$ equals 1. 


\section{Proof of the theorem.}

(4.1) We recall the Howe duality for unramified dual pairs.

The version of the Howe duality conjecture we use is the following: There exists a surjective homomorphism:

$$
\rho: \mathcal{H}\left(G O_{6} / / K^{\prime \prime}\right) \rightarrow \mathcal{H}\left(G S p_{2} / / K^{\prime}\right)
$$

which satisfies:

$$
\int_{G O_{6}} f\left(h^{-1}\right) \Omega(h, 1) \Phi_{0} d h=\int_{G S p_{2}} f^{\prime}\left(g^{\prime-1}\right) \Omega\left(1, g^{\prime}\right) \Phi_{0} d g^{\prime} .
$$

From now on, we assume the existence of the above homomorphism.

Let $f, f^{\prime}$ be Hecke functions of $G L_{4}$ and $G S p_{2}$. It follows from Prop. 7.1 of $[\mathbf{S a}]$ that there is an injective homomorphism $\iota: \mathcal{H}\left(G L_{4} / / K\right) \rightarrow$ $\mathcal{H}\left(G O_{6} / / K^{\prime \prime}\right)$. The composition of $\rho$ and $\iota$ is then a homomorphism from $\mathcal{H}\left(G L_{4} / / K\right)$ to $\mathcal{H}\left(G S p_{2} / / K^{\prime}\right)$. The functions $f$ and $f^{\prime}$ match if

$$
\rho(\iota(f))=f^{\prime} .
$$

It follows from the definition of $\iota$ and equation (18) that:

Theorem 2. If $f$ and $f^{\prime}$ are matching Hecke functions, then

$$
\int f\left(g^{-1}\right) \Omega(j(g), 1) \Phi_{0} d g=\int f^{\prime}\left(g^{\prime-1}\right) \Omega\left(1, g^{\prime}\right) \Phi_{0} d g^{\prime} .
$$

We will denote the LHS of (19) by $f *^{\prime} \Phi_{0}$ and the RHS by $f^{\prime} *^{\prime} \Phi_{0}$.

(4.2) We express the orbital integrals using the representation $\Omega$.

Lemma 3. If $f \in \mathcal{H}\left(G L_{4} / / K\right)$, let $\Phi=f *^{\prime} \Phi_{0}$, then $I_{f}(w, \mathbf{a})$ equals:

$\int_{N \cap w^{-1} H w \backslash N} \int \Omega(j(w \mathbf{a} n), t(u(z))) \Phi\left(a X_{0}, a^{-2}\right)|a|^{6} \chi(a) \psi(-z) \theta(n) d^{\times} a d z d n$.

Proof. Let $f_{1} * f_{2}$ denote the convolution of functions. Then $f * f_{0}=f$ if $f \in \mathcal{H}\left(G L_{4} / / K\right)$. The lemma follows from this fact and Lemma 1 .

Meanwhile it is easy to see that $J_{f^{\prime}}\left(w^{\prime}, \mathbf{t}\right)$ can be written into:

$$
\int_{N^{\prime}} \int_{N^{\prime} \cap w^{\prime-1} \bar{N}^{\prime} w^{\prime} \backslash N^{\prime}} \int f^{\prime}\left(n_{1}^{-1} w_{0}^{-1} w^{\prime} \mathbf{t} n_{2} \zeta\right) \theta^{\prime}\left(n_{1} n_{2}\right) \chi(\zeta) d^{\times} \zeta d n_{1} d n_{2} .
$$

Use Lemma 2 and the fact that $f^{\prime} * f_{0}^{\prime}=f^{\prime}$ for $f^{\prime} \in \mathcal{H}\left(G S p_{2} / / K^{\prime}\right)$, we get:

Lemma 4. If $f^{\prime} \in \mathcal{H}\left(G S p_{2} / / K^{\prime}\right)$, let $\Phi=f^{\prime} *^{\prime} \Phi_{0}$, then $J_{f^{\prime}}\left(w^{\prime}, \mathbf{t}\right)$ equals

$\int_{N^{\prime} \cap w^{\prime-1} \bar{N}^{\prime} w^{\prime} \backslash N^{\prime}} \iint \Omega\left(1, w_{0}^{-1} w^{\prime} \mathbf{t} n_{2} \zeta\right) \Phi\left(Y_{0}, 1\right) \psi(x+y) \theta^{\prime}\left(n_{2}\right) \chi(\zeta) d^{\times} \zeta d Y_{0} d n_{2}$. 
When $f$ and $f^{\prime}$ match, $f *^{\prime} \Phi_{0}=f^{\prime} *^{\prime} \Phi_{0}=\Phi$ for a function $\Phi$ satisfying the relation (16) and the relation:

$$
\Phi(Z, a)=0, a \notin \mathcal{O}^{\times} ; \Phi(z, a)=\Phi(z, 1), a \in \mathcal{O}^{\times} .
$$

Thus to prove the Theorem 1, we only need to show the following:

Lemma 5. For a function $\Phi$ on $M_{6,2}(F) \times F^{\times}$, satisfying (16) and (22), we have

$$
\chi(\operatorname{det}(w \mathbf{a}))^{-1 / 2} \times(20)=\chi\left(\lambda\left(w^{\prime} \mathbf{t}\right)\right)^{1 / 2} \times(21)
$$

when $(w, \mathbf{a})$ and $\left(w^{\prime}, \mathbf{t}\right)$ match.

In the rest of the section, we prove the equality $(23)$ for $(w, \mathbf{a})$ and $\left(w^{\prime}, \mathbf{t}\right)$ of type $(1),\left(1^{\prime}\right)$. The other cases are simpler and can be treated similarly. The proof is a simple computation using the properties of the representation $\Omega(\S 2)$ and the Fourier inversion formula. We note that in the case $(w, \mathbf{a})$ of type (1), $N \cap w^{-1} H w=\{1\}$; when $\left(w^{\prime}, \mathbf{t}\right)$ is of type $\left(1^{\prime}\right), N^{\prime} \cap w^{-1} \bar{N}^{\prime} w^{\prime}=$ $\{1\}$.

(4.3) We compute (20) for ( $w, \mathbf{a})$ of type (1).

From (10), the integral (20) equals:

$$
\iiint \Omega(j(n), t(u(z))) \Phi\left(a\left[\begin{array}{cc}
0 & 0 \\
0 & -a_{1}^{-1} a_{2}^{-1} \\
0 & 0 \\
0 & 0 \\
0 & -a_{1}^{-1} \\
1 & 0
\end{array}\right],-a^{-2} a_{1}^{2} a_{2}\right)
$$

From (22), we can restrict the domain of integration to where $\left|a_{1}^{2} a_{2}\right|=|a|^{2}$. Observe that $\chi(\operatorname{det}(w \mathbf{a}))=\chi\left(a_{1}^{2} a_{2}\right)$. Thus over the domain of integration, we have $\chi(\operatorname{det}(w \mathbf{a}))^{-1 / 2} \chi(a)=1$ and $|a|^{6}\left|a_{1}^{2} a_{2}\right|^{-3}=1$. Make a change of variable $a \rightarrow a a_{1} a_{2}$ and use (22), we get the LHS of (23) is:

$$
\int_{|a|^{2}=\left|a_{2}\right|^{-1}} \iint \Omega(j(n), t(u(z))) \Phi\left(a\left[\begin{array}{cc}
0 & 0 \\
0 & -1 \\
0 & 0 \\
0 & 0 \\
0 & -a_{2} \\
a_{1} a_{2} & 0
\end{array}\right], 1\right) \psi(-z) d^{\times} a d z \theta(n) d n .
$$

This is the expression we use to compare with the RHS of (23).

(4.4) We compute $(21)$ for $\left(w^{\prime}, \mathbf{t}\right)$ of type $\left(1^{\prime}\right)$. 
We can write $w_{0}^{-1} w^{\prime} \mathbf{t} n \zeta$ as

$$
\begin{aligned}
w_{0}^{-1} t\left(\left[\begin{array}{ll}
-a_{1} a_{2} & \\
& -a_{2}
\end{array}\right]\right)\left[\begin{array}{rr}
I & -a_{2}^{-1} V \\
& I
\end{array}\right]\left[\begin{array}{ll}
\zeta I & \\
& -\zeta a_{2} I
\end{array}\right] \\
\cdot t\left(\left[\begin{array}{ll}
1 & \\
& -1
\end{array}\right] u(-z)\left[\begin{array}{rr}
1 & \\
& -1
\end{array}\right]\right) .
\end{aligned}
$$

Then $\theta^{\prime}(n)=\psi\left(z+V_{2,1}\right)$ and $d n=d V d z$. We will implicitly use the fact that $\gamma(a, \psi)=1$ when the valuation of $a$ is even.

First consider the integration of (21) over $Y_{0}$. From (13), we have:

$$
\begin{aligned}
& \int \Omega\left(1, w_{0}^{-1} g\right) \Phi\left(Y_{0}, 1\right) \psi(x+y) d Y_{0} \\
& =\iint \Omega(1, g) \Phi(Z, 1) \psi\left(\operatorname{tr}\left({ }^{t} Z \tau_{6} Y_{0}\right)\right) \psi(x+y) d Y_{0} d Z .
\end{aligned}
$$

We can integrate over $Y_{0}$ and $Z_{16}, Z_{25}, Z_{26}$. From the Fourier inversion formula $\hat{\phi}(x)=\phi(-x)$, the above integral equals:

$$
\int \Omega(1, g) \Phi\left(\left[\begin{array}{cc}
Z_{11} & Z_{12} \\
\vdots & \vdots \\
Z_{15} & -1 \\
-1 & 0
\end{array}\right], 1\right) \psi\left(-Z_{23}-Z_{24}-Z_{15}\right) d Z .
$$

Apply Witt's Theorem, we can write this integral into:

$$
\iint_{N} \Omega(j(n), g) \Phi\left(\left[\begin{array}{cc}
\alpha & \beta \\
0 & \eta \\
0 & 0 \\
0 & 0 \\
0 & -1 \\
-1 & 0
\end{array}\right], 1\right) \theta(n) d n d(\alpha \beta \eta) .
$$

With the above consideration of the integration over $Y_{0}$, from (11), (12) and (16), we see (21) equals $\left|a_{1} a_{2}^{2}\right|^{3}$ times:

$$
\begin{aligned}
\iiint \iint \Omega\left(j(n),\left[\begin{array}{ll}
\zeta I & \\
& -\zeta a_{2} I
\end{array}\right] t\left(\left[\begin{array}{ll}
1 & \\
& -1
\end{array}\right] u(-z)\right)\right) \\
\cdot \Phi\left(\left[\begin{array}{cc}
-\alpha a_{1} a_{2} & -\beta a_{2} \\
0 & -\eta a_{2} \\
0 & 0 \\
0 & 0 \\
0 & a_{2} \\
a_{1} a_{2} & 0
\end{array}\right], 1\right)
\end{aligned}
$$

$\psi\left(\beta a_{1} a_{2} V_{1,2}+\alpha a_{1}^{2} a_{2} V_{1,1}+\eta a_{2} V_{2,1}+V_{2,1}\right) \psi(z) \theta(n) \chi(\zeta) d^{\times} \zeta d V d(\alpha \beta \eta) d n d z$. 
Use the Fourier inversion formula to integrate over $\alpha, \beta, \eta$ and $V$, we get (21) equals $\left|a_{2}\right|^{3}$ times:

$$
\begin{aligned}
\iiint \Omega\left(j(n),\left[\begin{array}{ll}
\zeta I & \\
& -\zeta a_{2} I
\end{array}\right] t\left(\left[\begin{array}{cc}
1 & \\
& -1
\end{array}\right] u(z)\right)\right) \\
\cdot \Phi\left(\left[\begin{array}{cc}
0 & 0 \\
0 & 1 \\
0 & 0 \\
0 & 0 \\
0 & a_{2} \\
a_{1} a_{2} & 0
\end{array}\right], 1\right) \psi(z) \theta(n) \chi(\zeta) d^{\times} \zeta d n d z .
\end{aligned}
$$

Change $z \rightarrow-z$, apply (12) and (14); we get (21) equals

$$
\begin{aligned}
& \left|a_{2}\right|^{3} \iiint|\zeta|^{6} \Omega(j(n), t(u(z))) \Phi\left(\zeta\left[\begin{array}{cc}
0 & 0 \\
0 & -1 \\
0 & 0 \\
0 & 0 \\
0 & -a_{2} \\
a_{1} a_{2} & 0
\end{array}\right],-a_{2}^{-1} \zeta^{-2}\right) \\
& \text { - } \psi(-z) \theta(n) \chi(\zeta) d^{\times} \zeta d n d z .
\end{aligned}
$$

From (22), we can restrict the domain of integration to where $\left|a_{2}\right|=|\zeta|^{-2}$. Over the domain, we have then

$$
\chi(\zeta) \chi\left(\lambda\left(w^{\prime} \mathbf{t}\right)\right)^{1 / 2}=\chi(\zeta) \chi\left(a_{2}\right)^{1 / 2}=1
$$

and $\left|a_{2}\right|^{3}|\zeta|^{6}=1$. Thus the RHS of (23), which equals $\chi\left(\lambda\left(w^{\prime} \mathbf{t}\right)\right)^{1 / 2} \times(25)$, is exactly (24). We have proved the Lemma 5 in the case when $(w, \mathbf{a})$ and $\left(w^{\prime}, \mathbf{t}\right)$ are of type $(1)$ and $\left(1^{\prime}\right)$.

\section{A generalization of Lemma 2 .}

Our method in proving Theorem 1 can be applied in other situations. The Lemmas that are stated here for $G L_{4}$ and $G S p_{2}$ can be generalized to other groups. We state the generalizations of Lemma 2 to the case of $S p_{n}, M p_{n}$. The proof for the Lemmas are similar to that of Lemma 2, and will be skipped. In the cases of $S p_{n}, M p_{n}$, we use the Weil representation $\omega$ of the groups $M p_{n(2 n+2)}$ and $M p_{n(2 n+1)}$ respectively. Let $N^{\prime}$ be the unit upper triangular subgroup of $S p_{n}$. The metaplectic group $M p_{n}$ splits over $N^{\prime}$, thus we can consider $N^{\prime}$ as a subgroup of $M p_{n}$ as well. In this case, we define a character $\theta^{\prime \prime}$ on $N^{\prime}$ by $\theta^{\prime \prime}(n)=\psi\left(n_{1,2}+\cdots+n_{n-1, n}+n_{n, n+1} / 2\right)$. Let $f_{0}^{\prime \prime}$ be the unit Hecke function of $M p_{n}$. 
Lemma 6. For $g \in S p_{n}$,

$$
\int_{N^{\prime}} f_{0}^{\prime}\left(n^{-1} g\right) \theta^{\prime}(n) d n=\int \omega(1, g) \Phi_{0}(X) \psi\left(X_{1,1}+\cdots+X_{n, n}\right) d X
$$

where $X \in M_{2 n+2, n}, X_{i+1, i}=X_{n+2, n}=-1, X_{j, i}=0$ for $1 \leq i \leq n, j>i+1$ (except when $j=n+2, i=n$ ), and other $X_{j, i}$ are free variables.

Lemma 7. For $g \in M p_{n}$,

$$
\int_{N^{\prime}} f_{0}^{\prime \prime}\left(n^{-1} g\right) \theta^{\prime \prime}(n) d n=\int \omega(1, g) \Phi_{0}(X) \psi\left(X_{1,1}+\cdots+X_{n, n}\right) d X
$$

where $X \in M_{2 n+1, n}, X_{i+1, i}=-1, X_{j, i}=0$ for $1 \leq i \leq n, j>i+1$, and other $X_{j, i}$ are free variables.

We expect these formulas to be useful in other cases of lifting. For example in $[\mathbf{M}]$, one needs to consider an orbital integral of the form

$$
\int_{N^{\prime}} \int_{N^{\prime} \cap g^{-1} N^{\prime} g \backslash N^{\prime}} f_{0}^{\prime \prime}\left(n_{1}^{-1} g n_{2}\right) \theta^{\prime \prime}\left(n_{1} n_{2}\right) d n_{1} d n_{2} .
$$

Then by applying Lemma 7, we can write the orbital integral using the Weil representation: It equals

$$
\iint_{N^{\prime} \cap g^{-1} N^{\prime} g \backslash N^{\prime}} \omega\left(1, g n_{2}\right) \Phi_{0}(X) \theta^{\prime \prime}\left(n_{2}\right) \psi\left(X_{1,1}+\cdots+X_{n, n}\right) d X d n_{2} .
$$

We can again use the properties of the Weil representation to study this integral.

\section{References}

[F-J] S. Friedberg and H. Jacquet, The fundamental lemma for the Shalika subgroup of $G L(4)$, Memoirs. AMS, 124(594) (1996).

[J] H. Jacquet, On the nonvanishing of some L-functions, Proc. Indian Acad. Sci., 97 (1987), 117-155.

[J-S] H. Jacquet and J. Shalika, Exterior square L-functions, in 'Automorphic Forms, Shimura Varieties and L-functions', Vol. II, Perspectives in Mathematics, 11 (1990), 143-226.

[K-S] D. Kazhdan and G. Savin, The smallest representation of simply laced groups, in 'Israel Math. Conf. Proc.', 2 (1990), 209-223.

[M] Z. Mao, Relative Salié sums, C.R. Acad. Sci. Paris, 316, Série I, (1993), 1257-1262.

[M-R] Z. Mao and S. Rallis, Howe duality and the trace formula, preprint.

[PS-S] I. Piatetski-Shapiro and D. Soudry, $L$ and $\epsilon$ factors for $G S p(4)$, J. Fac. Sci. Univ. Tokyo Sect. IA Math., 28 (1982), 505-530.

[R] S. Rallis, Langlands functoriality and the Weil representation. Amer. J. Math., 104(3) (1982), 469-515.

[Ro] B. Roberts, The theta correspondence for similitudes, Israel J. Math., 94 (1996), 285-317. 
[Sa] I. Satake, Theory of spherical functions on reductive algebraic groups over p-adic fields, Publ. Math. IHES, 18 (1963), 229-291.

[Wa] J.-L. Waldspurger, Demonstration d'une conjecture de duality de Howe dans le case $p$-adiques, $p \neq 2$, in 'Israel Math. Conf. Proc.', 2 (1990), 267-324.

[W] A. Weil, Sur certains groupes d'opérateurs unitaires, Acta Math, 111 (1964), 143211.

Received September 5, 1997 and revised September 24, 1998

Rutgers UNIVERSITY

NEWARK, NJ 07102-1811

E-mail address: zmao@andromeda.rutgers.edu

The Ohio State University

Columbus, OH 43210

E-mail address: haar@math.ohio-state.edu

The first author was partially supported by by NSF DMS 9304580 and the second author was partially supported by NSF DMS 7209098. 